Received: 23 January 2017

Accepted: 29 June 2017

Published online: 02 August 2017

\section{Neural substrates of embodied natural beauty and social endowed beauty: An fMRI study}

\author{
Wei Zhang ${ }^{1,2}$, Xianyou He ${ }^{1,3}$, Siyan Lai ${ }^{1}$, Juan Wan ${ }^{1}$, Shuxian Lai ${ }^{4}$, Xueru Zhao ${ }^{1}$ \& Darong $\mathrm{Li}^{1}$
}

What are the neural mechanisms underlying beauty based on objective parameters and beauty based on subjective social construction? This study scanned participants with fMRI while they performed aesthetic judgments on concrete pictographs and abstract oracle bone scripts. Behavioral results showed both pictographs and oracle bone scripts were judged to be more beautiful when they referred to beautiful objects and positive social meanings, respectively. Imaging results revealed regions associated with perceptual, cognitive, emotional and reward processing were commonly activated both in beautiful judgments of pictographs and oracle bone scripts. Moreover, stronger activations of orbitofrontal cortex (OFC) and motor-related areas were found in beautiful judgments of pictographs, whereas beautiful judgments of oracle bone scripts were associated with putamen activity, implying stronger aesthetic experience and embodied approaching for beauty were elicited by the pictographs. In contrast, only visual processing areas were activated in the judgments of ugly pictographs and negative oracle bone scripts. Results provide evidence that the sense of beauty is triggered by two processes: one based on the objective parameters of stimuli (embodied natural beauty) and the other based on the subjective social construction (social endowed beauty).

How people perceive beauty is the most important and debated issue in aesthetic psychology and neuroaesthetics. According to aesthetic theories, a sense of beauty may be mainly affected by two factors ${ }^{1}$. The first is objective parameters and external morphology of concrete objects, consistent with the perspectives of Plato's objectivist view of aesthetic perception ${ }^{1}$. Previous studies have found symmetrical human faces ${ }^{2-4}$, geometrical shapes ${ }^{5}$ and websites designs $s^{6}$, symmetrical sequences of apparent movements ${ }^{7}$, representational artworks ${ }^{8-10}$, sculptures obeying the canonical proportion of the golden ratio $^{1}$, and paintings and polygon patterns with intermediate complexity $^{11,12}$, typically elicit higher aesthetic appraisal and experience, providing support of this theory that "beauty has corresponding morphological characteristics".

An alternative theory argues that the perception of beauty is mainly based on the subjective construction of valence based on abstract social meaning and values. Prior studies have found smiling human faces ${ }^{13}$ with direct eye gaze $\mathrm{e}^{14}$, people with good inner character ${ }^{15-17}$, and short sentences ${ }^{18}$ and scenes ${ }^{19}$ describing morally-positive actions can elicit a sense of beauty and produce enhanced activation of aesthetic reward systems due to the positive social meanings associated with these stimuli. These studies imply that aesthetic evaluation is a subjective construction and support the "what is good is beautiful" theory.

Although there has been progress in the neuroimaging of aesthetic appraisal, very little is known regarding the mental and neural mechanisms underlying these seemingly contradictory theories. Previous studies have found that ancient Chinese characters can arouse a sense of beauty due to their obvious two-dimensional graphical features ${ }^{20,21}$, supporting the use of ancient Chinese characters as materials for studies of aesthetic appraisal and judgments. More importantly, ancient Chinese characters were produced using two separate systems, one in which characters referring to concrete objects were generated by outlining the shape of the object (pictographs), and another in which characters referring to abstract social meaning were developed to convey corresponding

${ }^{1}$ Guangdong Key Laboratory of Mental Health and Cognitive Science, Center for Studies of Psychological Application, School of Psychology, South China Normal University, Guangzhou, P. R. China. ${ }^{2}$ School of Architecture, Guangdong Engineering \& Technology Research Center for Modern Architecture Design, State Key Laboratory of Subtropical Building Science, South China University of Technology, Guangzhou, P. R. China. ${ }^{3}$ Key Laboratory of Chinese Learning and International Promotion of South China Normal University, Guangzhou, P. R. China. ${ }^{4}$ Public Kindergarten of Guangzhou Government, Guangzhou, P. R. China. Wei Zhang and Xianyou He contributed equally to this work. Correspondence and requests for materials should be addressed to X.H. (email: xianyouhe@163.com) 
social concepts (ideographic symbol of oracle bone scripts) ${ }^{22,23}$. Aesthetic qualities of pictographs and oracle bone script therefore may be based on the beauty of objective morphologies and the beauty of subjective social construction, respectively.

Following the logic of the studies examining aesthetic perception and appraisal of pictographs ${ }^{20,21}$, we asked the question of whether oracle bone scripts would elicit aesthetic appraisal dependent on their abstract social meanings. That is, would oracle bone scripts that refer to positive social meanings be more likely to be judged as beautiful, and those that refer to negative social meanings be more likely to be judged as ugly? The answer to this question may contribute to identifying the mental and neural mechanisms underlying the beauty of objective morphologies and the beauty of subjective social construction.

The present functional magnetic resonance imaging (fMRI) study investigated the neural basis of aesthetic appraisal of objective morphologies and subjective social constructions when participants observed and made aesthetic judgments about ancient Chinese characters referring to concrete objects (aesthetic judgment of pictograph, AP) and abstract social meanings (aesthetic judgment of oracle bone script, AO). The effect of semantic processing during the aesthetic judgments was prevented by using pictographs and oracle bone scripts that were unfamiliar to participants. In addition, high or low luminance grey squares were used in a baseline task (square luminance judgment, SL) to control for activity in motor brain regions associated with the key responses. The neural substrates of pictograph aesthetic judgments were identified by the contrast of "AP $>$ SL", and the neural substrates of oracle bone script aesthetic judgments were identified by the contrast of "AO $>$ SL". According to our hypothesis, if pictographs and oracle bone scripts elicit variable aesthetic appraisals related to the aesthetic qualities of the referential objects and the positive degrees of the referential social meanings, respectively, then similar neural areas should be engaged in both tasks, including regions of the fusiform gyrus, occipital gyri, inferior and middle frontal gyri, anterior cingulate cortex, OFC, caudate nucleus and putamen which are associated with perceptual, cognitive, emotional and reward processing ${ }^{24-26}$.

Because pictographs reflect the shapes of their referential objects, they have a higher degree of visualization than oracle bone scripts referring to abstract meanings. We hypothesized that this high visualization for pictographs would activate perceptual representations, and produce relevant image schemas and visual mental images which could serve to elicit strong recurring bodily experiences ${ }^{27}$. These bodily experiences might result in an embodied contribution towards beauty, which can be regarded as bodily resonance for the intention of making action toward beauty ${ }^{21,28,29}$, and which should be revealed by stronger activations in motor-related regions ${ }^{21,28}$.

\section{Results}

Behavioral results. Judgments of Beauty. Judgments of beauty were used as behavioral dependent variable, calculated as the rates at which pictographs or oracle bone scripts were judged to be beautiful. A 2 (type of referent: pictograph referring to concrete objects vs. oracle bone script referring to abstract social meanings) $\times 2$ (valence of referent: positive vs. negative) repeated-measures ANOVA revealed a significant main effect of type of referent, $F(1,16)=64.28, p<0.001, M S e=0.02, \eta^{2}=0.80$. A significant main effect of valence of referent was also found, $F(1,16)=338.07, p<0.001, M S e=0.01, \eta^{2}=0.96$, as well as a significant interaction between type of referent and valence of referent, $F(1,16)=8.83, p<0.010, M S e=0.01, \eta^{2}=0.36$.

The simple effect of beauty for the pictographs revealed higher ratings when pictographs referred to beautiful objects $(0.73 \pm 0.13)$ than when they referred to ugly objects $(0.32 \pm 0.13), F(1,16)=90.98, p<0.001$, $\eta^{2}=0.85$. Likewise, oracle bone scripts were rated higher in beauty when they referred to positive social meanings $(0.70 \pm 0.12)$ than to negative social meanings $(0.17 \pm 0.08), F(1,16)=729.54, p<0.001, \eta^{2}=0.98$. There was no significant difference in beauty judgment between pictographs that referred to beautiful objects and oracle bone scripts that referred to positive social meanings, $F(1,16)=1.44, p=0.248, \eta^{2}=0.08$. However, the rate of beautiful judgments for pictographs that referred to ugly objects was higher than that for oracle bone scripts that referred to negative social meanings, $F(1,16)=39.10, p<0.001, \eta^{2}=0.71$, suggesting that viewing pictographs and oracle bone scripts could differentially arouse the sense of beauty depending on the valence of their referents.

Post-scan ratings. The mean ratings performed post-scanning showed participants overall reported that it was difficult to guess the semantic meaning of any of the characters. Low ratings held across character and valence, including pictographs referring to beautiful objects $(1.76 \pm 0.32)$ and ugly objects $(1.57 \pm 0.32)$, and oracle bone scripts referring to positive $(1.72 \pm 0.45)$ and negative social meanings $(1.39 \pm 0.29)$. These consistently low ratings suggest that it was unlikely that participants based their aesthetic judgments of the four sets of stimuli on their semantic meanings.

fMRI results. Brain regions for aesthetic judgments of pictographs with aesthetic valence. In order to identify brain regions sensitive to aesthetic valence of pictographs, the "PB $>\mathrm{HL}$ " and "PU $>$ LL" contrasts were examined. Similar to the findings of Zhang et al. ${ }^{21}$, brain activity related to the contrast of "PB $>\mathrm{HL}$ " was found in large-scale brain networks, including the bilateral inferior occipital gyri, left middle occipital gyrus, bilateral inferior frontal gyri, left medial superior frontal gyrus, right inferior OFC, left hippocampus, left superior parietal lobule, right supramarginal gyrus extending to postcentral gyrus, and right paracentral lobule (see Table 1 and Fig. 1a). Only bilateral inferior occipital gyri were activated in the contrast of "PU > LL" (see Table 1 and Fig. 1b).

Brain regions for aesthetic judgments of oracle bone scripts with aesthetic valence. In the contrast of "OP $>\mathrm{HL}$ ", we found that beauty judgments of oracle bone scripts referring to positive social meanings were associated with activity in the bilateral inferior occipital gyri and inferior frontal gyri, left medial superior frontal gyrus, bilateral hippocampus, and right putamen (see Table 2, Fig. 2a). For the contrast of "ON $>$ LL", we observed significantly stronger activations in the bilateral inferior occipital gyri and right middle occipital gyrus (see Table 2, Fig. 2b). 


\begin{tabular}{|c|c|c|c|c|c|c|}
\hline \multirow[b]{2}{*}{ Brain regions } & \multirow[b]{2}{*}{ Hemisphere } & \multicolumn{3}{|c|}{ Peak coordinates } & \multirow[b]{2}{*}{$t$-score } & \multirow{2}{*}{$\begin{array}{l}\text { Cluster } \\
\text { size }\end{array}$} \\
\hline & & $\mathbf{x}$ & $\mathbf{y}$ & $\mathbf{z}$ & & \\
\hline \multicolumn{7}{|c|}{ Beautiful pictograph $>$ High luminance } \\
\hline \multirow{2}{*}{ Inferior occipital gyrus } & $\mathrm{L}$ & -33 & -90 & -9 & 17.70 & 1736 \\
\hline & $\mathrm{R}$ & 24 & -99 & -6 & 19.51 & 2309 \\
\hline Middle occipital gyrus & $\mathrm{L}$ & -24 & -72 & 30 & 5.73 & 369 \\
\hline \multirow{2}{*}{ Inferior frontal gyrus } & $\mathrm{L}$ & -48 & 30 & 15 & 7.50 & 827 \\
\hline & $\mathrm{R}$ & 45 & 12 & 27 & 7.27 & 611 \\
\hline Medial superior frontal gyrus & $\mathrm{L}$ & -6 & 21 & 42 & 5.26 & 195 \\
\hline Inferior OFC & $\mathrm{R}$ & 24 & 30 & -18 & 5.23 & 67 \\
\hline Hippocampus & $\mathrm{L}$ & -21 & -33 & 0 & 4.50 & 50 \\
\hline Superior parietal lobule & $\mathrm{L}$ & -24 & -63 & 48 & 5.31 & 369 \\
\hline $\begin{array}{l}\text { Supramarginal gyrus extending to } \\
\text { postcentral gyrus }\end{array}$ & $\mathrm{R}$ & 39 & -30 & 39 & 4.57 & 77 \\
\hline Paracentral lobule & $\mathrm{R}$ & 9 & -24 & 75 & 4.33 & 32 \\
\hline \multicolumn{7}{|l|}{ Ugly pictograph > Low luminance } \\
\hline \multirow{2}{*}{ Inferior occipital gyrus } & $\mathrm{L}$ & -33 & -90 & -9 & 13.59 & 638 \\
\hline & $\mathrm{R}$ & 24 & -99 & -6 & 14.24 & 763 \\
\hline
\end{tabular}

Table 1. Activated areas correlating with the aesthetic judgments of pictographs. Note: Coordinates refer to the stereotactic space of the Montreal Neurological Institute. The statistical significance refers to $p<0.001$ at voxel level (uncorrected), $p<0.05$ at cluster level (FWE corrected).

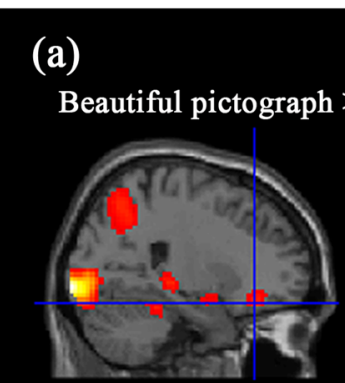

R. Inferior OFC
High luminance

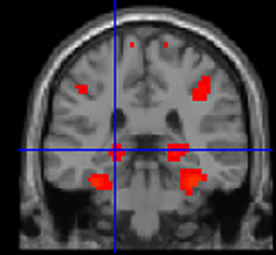

L. Hippocampus

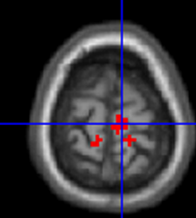

R. Paracentral lobule

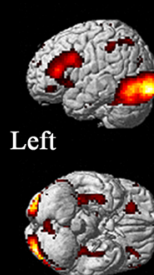

Ventral

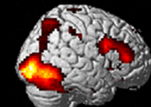

Right

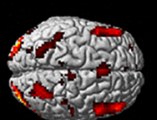

Dorsal (b)

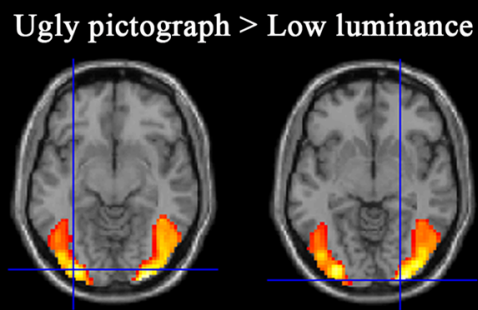

Bilateral IOG

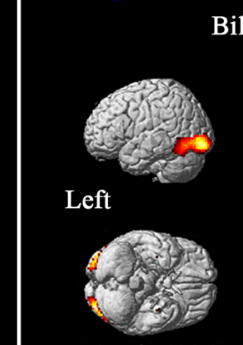

Ventral

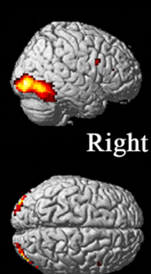

Dorsal

Figure 1. The main cerebral areas for aesthetic judgments of pictographs.

Brain regions revealed by conjunction analysis. Conjunction analyses were performed to identify brain activations common to aesthetic judgments of pictographs and oracle bone scripts. Results showed that bilateral inferior occipital gyri, left middle occipital gyrus, bilateral inferior frontal gyri, left medial superior frontal gyrus, left hippocampus and right inferior OFC were commonly activated when making beauty judgments for both pictographs and oracle bone scripts (see Table 3 and Fig. 3a). Moreover, conjunction analysis revealed common activation of bilateral inferior occipital gyri during judgments of both pictographs that referred to ugly objects and oracle bone scripts that referred to negative social meanings (see Table 3 and Fig. 3b).

Aesthetic cortical differentiation between pictographs and oracle bone scripts. In order to investigate the cortical differentiation of the aesthetic judgments between pictographs and oracle bone scripts, direct pairwise comparisons were made between the four sets of experimental materials within valence. We found enhanced activation of right cuneus and left inferior temporal gyrus in the contrast of "PB $>$ OP", but no significant differences were found in the opposite contrast. For the comparisons between pictographs referred to ugly objects and oracle bone scripts referred to negative social meanings, only the contrast of "ON $>$ PU" revealed stronger activations in left 


\begin{tabular}{|c|c|c|c|c|c|c|}
\hline \multirow[b]{2}{*}{ Brain regions } & \multirow[b]{2}{*}{ Hemisphere } & \multicolumn{3}{|c|}{ Peak coordinates } & \multirow[b]{2}{*}{$t$-score } & \multirow[b]{2}{*}{ Cluster size } \\
\hline & & $\mathbf{x}$ & $\mathbf{y}$ & $\mathbf{z}$ & & \\
\hline \multicolumn{7}{|c|}{ Positive oracle bone script $>$ High luminance } \\
\hline \multirow{2}{*}{ Inferior occipital gyrus } & $\mathrm{L}$ & -33 & -90 & -9 & 18.60 & 2046 \\
\hline & $\mathrm{R}$ & 24 & -99 & -6 & 18.30 & 2270 \\
\hline \multirow{2}{*}{ Inferior frontal gyrus } & $\mathrm{L}$ & -48 & 12 & 30 & 7.09 & 778 \\
\hline & $\mathrm{R}$ & 45 & 12 & 27 & 8.16 & 756 \\
\hline $\begin{array}{l}\text { Medial superior frontal } \\
\text { gyrus }\end{array}$ & $\mathrm{L}$ & -6 & 21 & 42 & 5.18 & 269 \\
\hline \multirow{2}{*}{ Hippocampus } & $\mathrm{L}$ & -33 & -6 & -27 & 5.45 & 234 \\
\hline & $\mathrm{R}$ & 21 & -33 & 0 & 5.39 & 192 \\
\hline Putamen & $\mathrm{R}$ & 27 & -6 & 12 & 4.02 & 21 \\
\hline \multicolumn{7}{|c|}{ Negative oracle bone script $>$ Low luminance } \\
\hline \multirow{2}{*}{ Inferior occipital gyrus } & $\mathrm{L}$ & -33 & -90 & -9 & 15.89 & 933 \\
\hline & $\mathrm{R}$ & 24 & -99 & -6 & 16.58 & 1000 \\
\hline Middle occipital gyrus & $\mathrm{R}$ & 30 & -66 & 36 & 6.50 & 132 \\
\hline
\end{tabular}

Table 2. Activated areas correlating with the aesthetic judgments of oracle bone scripts. Note: Coordinates refer to the stereotactic space of the Montreal Neurological Institute. The statistical significance refers to $p<0.001$ at voxel level (uncorrected), $p<0.05$ at cluster level (FWE corrected).

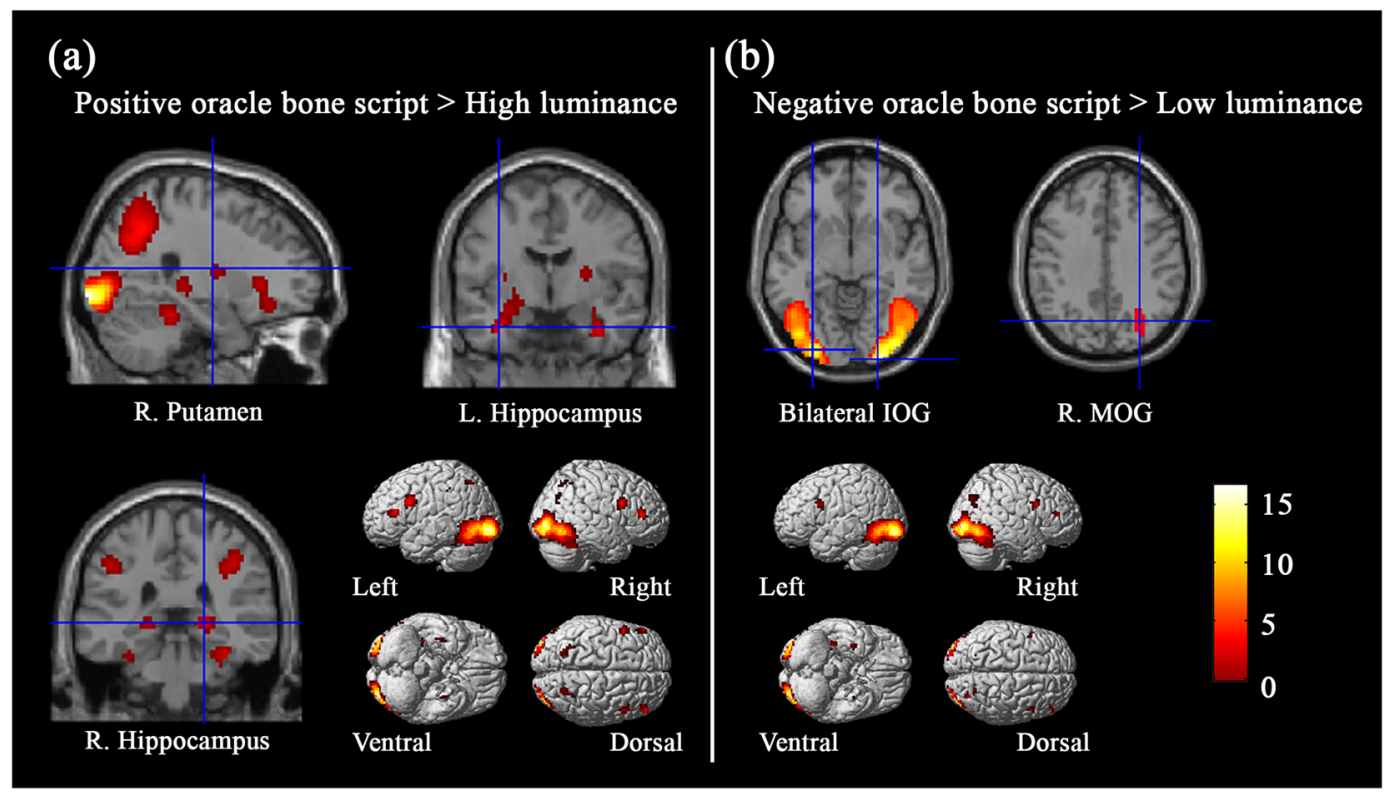

Figure 2. The main cerebral areas for aesthetic judgments of oracle bone scripts.

superior temporal gyrus and bilateral fusiform gyri, but there was no significant difference in the opposite contrast of "PU > ON" (see Table 4).

Cortical differentiation between beautiful and ugly stimuli. The contrast between pictographs that referred to beautiful objects and those that referred to ugly objects revealed stronger activations for pictographs referred to beautiful objects $(\mathrm{PB}>\mathrm{PU})$ in the left inferior temporal gyrus and cuneus, right calcarine sulcus and lingual gyrus. The opposite contrast of "PU $>\mathrm{PB}$ " revealed no significant activations. For the oracle bone scripts, no significant activations were found in the contrast of "OP $>\mathrm{ON}$ ", but stronger activations in the opposite contrast of "ON > OP" were observed in bilateral lingual gyri, left fusiform gyrus, right precentral gyrus, left middle temporal gyrus and paracentral lobule (see Table 5).

Brain regions for aesthetic judgments regardless of the aesthetic valence. To further understand the brain regions involved in aesthetic processing of concrete pictographs and abstract oracle bone scripts per se, we analyzed fMRI activity in the comparisons for the aesthetic judgment vs. baseline condition. Contrast of "AP $>$ SL" revealed significant activation in the bilateral inferior occipital gyri, left medial superior frontal gyrus, right inferior OFC, left hippocampus, right middle cingulum, right supramarginal gyrus, and right paracentral lobule (see Table 6). In the contrast of "AO $>$ SL", we found stronger activations in the bilateral inferior occipital gyri, bilateral inferior 


\begin{tabular}{|c|c|c|c|c|c|c|}
\hline \multirow[b]{2}{*}{ Brain regions } & \multirow[b]{2}{*}{ Hemisphere } & \multicolumn{3}{|c|}{ Peak coordinates } & \multirow[b]{2}{*}{$t$-score } & \multirow[b]{2}{*}{ Cluster size } \\
\hline & & $\mathbf{x}$ & y & $\mathbf{z}$ & & \\
\hline \multicolumn{7}{|c|}{ Conjunction of beautiful pictograph and positive oracle bone script } \\
\hline \multirow{2}{*}{ Inferior occipital gyrus } & $\mathrm{L}$ & -33 & -90 & -9 & 17.70 & 1398 \\
\hline & $\mathrm{R}$ & 24 & -99 & -6 & 18.30 & 1957 \\
\hline Middle occipital gyrus & $\mathrm{L}$ & -24 & -72 & 30 & 5.73 & 356 \\
\hline \multirow{2}{*}{ Inferior frontal gyrus } & $\mathrm{L}$ & -48 & 9 & 30 & 6.78 & 676 \\
\hline & $\mathrm{R}$ & 45 & 12 & 27 & 7.27 & 563 \\
\hline $\begin{array}{l}\text { Medial superior frontal } \\
\text { gyrus }\end{array}$ & $\mathrm{L}$ & -6 & 21 & 42 & 5.26 & 160 \\
\hline Hippocampus & $\mathrm{L}$ & -33 & -6 & -27 & 5.92 & 61 \\
\hline Inferior OFC & $\mathrm{R}$ & 27 & 30 & -12 & 4.42 & 34 \\
\hline \multicolumn{7}{|c|}{ Conjunction of ugly pictograph and negative oracle bone script } \\
\hline \multirow{2}{*}{ Inferior occipital gyrus } & $\mathrm{L}$ & -33 & -90 & -9 & 13.59 & 638 \\
\hline & $\mathrm{R}$ & 24 & -99 & -6 & 14.24 & 762 \\
\hline
\end{tabular}

Table 3. Activations in the conjunction analysis between pictographs and oracle bone scripts. Note: Coordinates refer to the stereotactic space of the Montreal Neurological Institute. The statistical significance refers to $p<0.001$ at voxel level (uncorrected), $p<0.05$ at cluster level (FWE corrected).

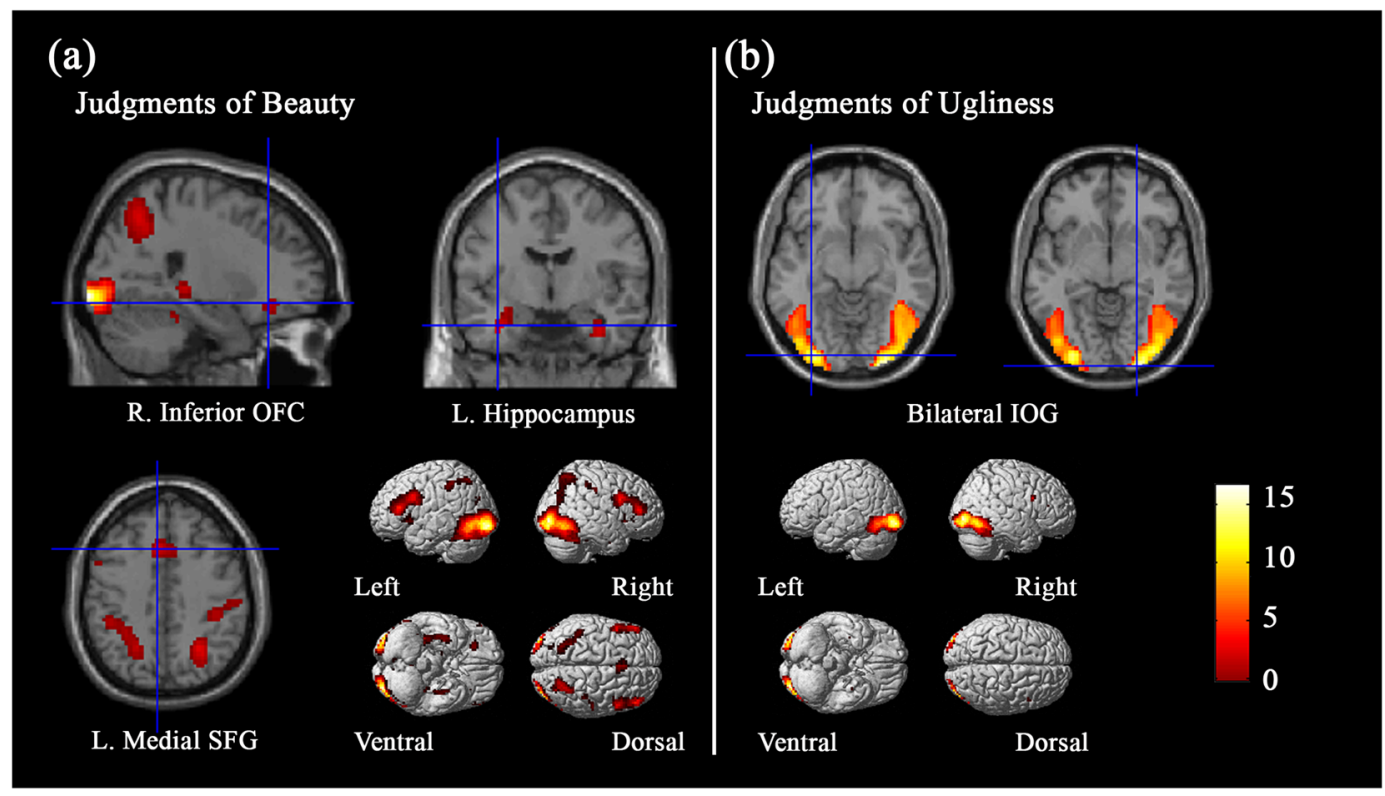

Figure 3. The conjunction results of the judgments of beautiful items and ugly items across pictographs and oracle bone scripts.

frontal gyri, left medial superior frontal gyrus, right inferior OFC, and left hippocampus (see Table 6). Meanwhile, we also computed a conjunction analyses between the "AP $>$ SL" and "AO $>$ SL". The results showed that bilateral inferior occipital gyri, left medial superior frontal gyrus, right middle cingulum, left hippocampus, right inferior OFC, and right supramarginal gyrus were commonly activated (see Table 7 and Fig. 4c).

To identify regions sensitive to the differences between pictographs and oracle bone scripts, we conducted two contrasts between conditions "AP" and "AO". In the contrast of "AP $>A O$ ", we found only right cuneus was activated (see Table 7 and Fig. 4a). Stronger activations were found in the left middle temporal gyrus, left superior parietal lobule, and left thalamus in the opposite contrast of "AO > AP" (see Table 7 and Fig. 4b).

\section{Discussion}

In this present study, we identified the neural basis underlying the aesthetic appraisal of ancient Chinese characters differing in referent (concrete objects versus abstract social concepts) and valence (positive and negative). As hypothesized, the behavioral data indicated that pictographs referring to beautiful concrete objects and oracle bone scripts referring to positive social meanings were more likely to be judged as beautiful. In contrast, pictographs referring to ugly objects and oracle bone scripts referring to negative social meanings were more likely to be judged as ugly, showing that the experimental materials used in the present study are capable of inducing aesthetic experience and appraisal. 


\begin{tabular}{|c|c|c|c|c|c|c|}
\hline \multirow[b]{2}{*}{ Brain regions } & \multirow[b]{2}{*}{ Hemisphere } & \multicolumn{3}{|c|}{ Peak coordinates } & \multirow[b]{2}{*}{$t$-score } & \multirow{2}{*}{$\begin{array}{l}\text { Cluster } \\
\text { size }\end{array}$} \\
\hline & & $\mathbf{x}$ & $\mathbf{y}$ & $\mathbf{z}$ & & \\
\hline \multicolumn{7}{|c|}{ Beautiful pictograph $>$ Positive oracle bone script } \\
\hline Cuneus & $\mathrm{R}$ & 12 & -93 & -18 & 4.52 & 288 \\
\hline Inferior temporal gyrus & $\mathrm{L}$ & -60 & -24 & -18 & 4.05 & 50 \\
\hline \multicolumn{7}{|c|}{ Positive oracle bone script $>$ Beautiful pictograph } \\
\hline \multicolumn{7}{|l|}{ Non-significant } \\
\hline \multicolumn{7}{|c|}{ Ugly pictograph $>$ Negative oracle bone script } \\
\hline \multicolumn{7}{|l|}{ Non-significant } \\
\hline \multicolumn{7}{|c|}{ Negative oracle bone script $>$ Ugly pictograph } \\
\hline Superior temporal gyrus & $\mathrm{L}$ & -57 & -3 & -6 & 4.20 & 72 \\
\hline \multirow{2}{*}{ Fusiform gyrus } & $\mathrm{L}$ & -27 & -54 & -9 & 4.18 & 39 \\
\hline & $\mathrm{R}$ & 33 & -39 & -15 & 4.04 & 103 \\
\hline
\end{tabular}

Table 4. Cortical networks of the analysis of variance between pictographs and oracle bone scripts. Note: Coordinates refer to the stereotactic space of the Montreal Neurological Institute. The statistical significance refers to $p<0.001$ at voxel level (uncorrected), $p<0.05$ at cluster level (FWE corrected).

\begin{tabular}{|c|c|c|c|c|c|c|}
\hline \multirow[b]{2}{*}{ Brain regions } & \multirow[b]{2}{*}{ Hemisphere } & \multicolumn{3}{|c|}{ Peak coordinates } & \multirow[b]{2}{*}{$t$-score } & \multirow{2}{*}{$\begin{array}{l}\text { Cluster } \\
\text { size }\end{array}$} \\
\hline & & $\mathbf{x}$ & $\mathbf{y}$ & $\mathbf{z}$ & & \\
\hline \multicolumn{7}{|c|}{ Beautiful pictograph $>$ Ugly pictograph } \\
\hline Inferior temporal gyrus & $\mathrm{L}$ & -57 & -54 & -15 & 4.60 & 48 \\
\hline Cuneus & $\mathrm{L}$ & 0 & -90 & 18 & 3.59 & 48 \\
\hline Calcarine sulcus & $\mathrm{R}$ & 12 & -66 & 15 & 4.57 & 169 \\
\hline Lingual gyrus & $\mathrm{R}$ & 18 & -99 & -6 & 4.41 & 36 \\
\hline \multicolumn{7}{|c|}{ Ugly pictograph $>$ Beautiful pictograph } \\
\hline \multicolumn{7}{|l|}{ Non-significant } \\
\hline \multicolumn{7}{|c|}{ Positive oracle bone script $>$ Negative oracle bone script } \\
\hline \multicolumn{7}{|l|}{ Non-significant } \\
\hline \multicolumn{7}{|c|}{ Negative oracle bone script $>$ Positive oracle bone script } \\
\hline \multirow{2}{*}{ Lingual gyrus } & $\mathrm{L}$ & -27 & -51 & -6 & 4.43 & 34 \\
\hline & $\mathrm{R}$ & 21 & -72 & -6 & 3.78 & 29 \\
\hline Fusiform gyrus & $\mathrm{L}$ & -27 & -75 & -6 & 3.76 & 34 \\
\hline Precentral gyrus & $\mathrm{R}$ & 12 & -21 & 75 & 4.34 & 20 \\
\hline Middle temporal gyrus & $\mathrm{L}$ & -51 & 6 & -18 & 4.14 & 34 \\
\hline Paracentral lobule & $\mathrm{L}$ & -6 & -33 & 57 & 4.00 & 21 \\
\hline
\end{tabular}

Table 5. Cortical networks of the analysis of variance between beautiful and ugly stimuli. Note: Coordinates refer to the stereotactic space of the Montreal Neurological Institute. The statistical significance refers to $p<0.001$ at voxel level (uncorrected), $p<0.05$ at cluster level (FWE corrected).

In line with previous findings, the bilateral inferior occipital gyri, bilateral inferior frontal gyri, left medial superior frontal gyrus, left hippocampus and right inferior OFC were activated during the aesthetic judgments (analysis regardless of aesthetic valence) and beautiful font structure judgments (analysis with aesthetic valence) of pictographs ${ }^{21}$. Prior findings have indicated that occipital gyrus plays an important part in early visual processing such as perception of shape and color $^{30}$, as well as visual attention to the beautiful visual stimuli ${ }^{21,31,32}$. The inferior frontal gyrus is also active in the aesthetic judgments of rhetorical beauty and moral beauty ${ }^{33}$, and this activation is considered to represent emotional states regardless of the actual affective or sensory modality ${ }^{34}$. Meanwhile, the superior frontal gyrus is related to aesthetic judgment and social cognition ${ }^{5,35}$, and the hippocampus is known for a functional correlation between processing of novel stimuli and evaluation of aesthetic and pleasant stimuli ${ }^{36-39}$. The OFC has been suggested to be important for aesthetic perception and judgment, and is reliably activated during both aesthetic appraisal and non-aesthetic appraisal tasks ${ }^{19}$, 40 . Previous studies also proposed that the OFC plays a key role in emotion, and is involved in the functional integration of emotion and cognition $^{41,42}$, which may be present in aesthetic emotion ${ }^{40}$.

More importantly, previous neuroimaging evidence suggests the sense of beauty may heighten spatial cognitive processes, increase somatosensory perception, and elicit the planning and intention of movements ${ }^{43}$. Our results support prior findings in which stronger activation of superior parietal lobule, right supramarginal gyrus extending to postcentral gyrus, and right paracentral lobule were found during the aesthetic judgments of pictographs. For instance, the superior parietal lobule is related to the visuo-spatial exploration and motor mapping ${ }^{32,44}$, supramarginal gyrus extending to postcentral gyrus is part of somatosensory association cortex ${ }^{45,46}$, and paracentral lobule is concerned with motor function and is often called the supplementary motor area ${ }^{47-49}$. Since we 


\begin{tabular}{|c|c|c|c|c|c|c|}
\hline \multirow[b]{2}{*}{ Brain regions } & \multirow[b]{2}{*}{ Hemisphere } & \multicolumn{3}{|c|}{ Peak coordinates } & \multirow[b]{2}{*}{$t$-score } & \multirow{2}{*}{$\begin{array}{l}\text { Cluster } \\
\text { size }\end{array}$} \\
\hline & & $\mathbf{x}$ & $\mathbf{y}$ & $\mathbf{z}$ & & \\
\hline \multicolumn{7}{|c|}{ Aesthetic judgments of pictograph $>$ Luminance judgments } \\
\hline \multirow[t]{2}{*}{ Inferior occipital gyrus } & $\mathrm{L}$ & -33 & -90 & -9 & 21.98 & 3289 \\
\hline & $\mathrm{R}$ & 24 & -99 & -6 & 23.68 & 2702 \\
\hline Medial superior frontal gyrus & $\mathrm{L}$ & -6 & 21 & 42 & 6.15 & 393 \\
\hline Inferior OFC & $\mathrm{R}$ & 27 & 33 & -18 & 9.15 & 892 \\
\hline Hippocampus & $\mathrm{L}$ & -21 & -33 & 0 & 5.04 & 68 \\
\hline Middle cingulum & $\mathrm{R}$ & 6 & 0 & 32 & 4.79 & 37 \\
\hline Supramarginal gyrus & $\mathrm{R}$ & 39 & -33 & 42 & 4.71 & 80 \\
\hline Paracentral lobule & $\mathrm{R}$ & 6 & -24 & 75 & 4.49 & 33 \\
\hline \multicolumn{7}{|c|}{ Aesthetic judgments of oracle bone script $>$ Luminance judgments } \\
\hline \multirow{2}{*}{ Inferior occipital gyrus } & $\mathrm{L}$ & -33 & -90 & -9 & 24.38 & 1631 \\
\hline & $\mathrm{R}$ & 24 & -99 & -6 & 24.65 & 1932 \\
\hline \multirow{2}{*}{ Inferior frontal gyrus } & $\mathrm{L}$ & -48 & 9 & 30 & 8.71 & 457 \\
\hline & $\mathrm{R}$ & 45 & 12 & 27 & 10.12 & 220 \\
\hline Medial superior frontal gyrus & $\mathrm{L}$ & -6 & 21 & 42 & 6.88 & 137 \\
\hline Inferior OFC & $\mathrm{R}$ & 27 & 30 & -12 & 5.77 & 21 \\
\hline Hippocampus & $\mathrm{L}$ & -33 & -6 & -27 & 7.74 & 60 \\
\hline
\end{tabular}

Table 6. Brain regions for aesthetic judgments of pictographs and oracle bone scripts regardless of the aesthetic valence. Note: Coordinates refer to the stereotactic space of the Montreal Neurological Institute. The statistical significance refers to $p<0.001$ at voxel level (uncorrected), $p<0.05$ at cluster level (FWE corrected).

\begin{tabular}{|c|c|c|c|c|c|c|}
\hline \multirow[b]{2}{*}{ Brain regions } & \multirow[b]{2}{*}{ Hemisphere } & \multicolumn{3}{|c|}{ Peak coordinates } & \multirow[b]{2}{*}{$t$-score } & \multirow{2}{*}{$\begin{array}{l}\text { Cluste } \\
\text { size }\end{array}$} \\
\hline & & $\mathbf{x}$ & $\mathbf{y}$ & $\mathbf{z}$ & & \\
\hline \multicolumn{7}{|c|}{ Conjunction of "AP $>$ SL" and "AO $>$ SL" } \\
\hline \multirow{2}{*}{ Inferior occipital gyrus } & $\mathrm{L}$ & -33 & -90 & -9 & 21.98 & 3105 \\
\hline & $\mathrm{R}$ & 24 & -99 & -6 & 23.68 & 2613 \\
\hline Medial superior frontal gyrus & $\mathrm{L}$ & -6 & 21 & 42 & 6.15 & 303 \\
\hline Middle cingulum & $\mathrm{R}$ & 6 & 0 & 32 & 4.79 & 32 \\
\hline Hippocampus & $\mathrm{L}$ & -21 & -33 & 0 & 5.04 & 61 \\
\hline Inferior OFC & $\mathrm{R}$ & 27 & 30 & -15 & 5.69 & 850 \\
\hline Supramarginal gyrus & $\mathrm{R}$ & 39 & -33 & 42 & 4.71 & 79 \\
\hline \multicolumn{7}{|c|}{ Aesthetic judgments of pictograph $>$ Aesthetic judgments of oracle bone script } \\
\hline Cuneus & $\mathrm{R}$ & 12 & -93 & 15 & 4.04 & 13 \\
\hline \multicolumn{7}{|c|}{ Aesthetic judgments of oracle bone script $>$ Aesthetic judgments of pictograph } \\
\hline Middle temporal gyrus & $\mathrm{L}$ & -48 & -69 & 9 & 4.61 & 96 \\
\hline Superior parietal lobule & $\mathrm{L}$ & -24 & -69 & 60 & 3.52 & 20 \\
\hline Thalamus & $\mathrm{L}$ & -15 & -15 & -6 & 3.83 & 21 \\
\hline
\end{tabular}

Table 7. Cortical networks of the analysis of conjunction, and the analysis of variance between pictographs and oracle bone scripts regardless of the aesthetic valence. Note: Coordinates refer to the stereotactic space of the Montreal Neurological Institute. The statistical significance refers to $p<0.001$ at voxel level (uncorrected), $p<0.05$ at cluster level (FWE corrected).

adopted the square luminance judgment task as a baseline task to control for the activation of motor-related areas in response to key pressing, we infer that visuo-spatial exploration and motor representation may be associated with aesthetic orientation. More precisely, these motor-related regions may be implicated in the generation of embodied approach and motivation for beauty, and the above neural results could be interpreted as indicating that beauty judgments of pictographs involve joint participation of perceptual processing, cognitive judgment, reward and embodied experience ${ }^{21,28,29}$.

For both the aesthetic judgments overall and beautiful judgments specifically of oracle bone scripts, neuroimaging results revealed similar neural regions including bilateral inferior occipital gyri, bilateral inferior frontal gyri, left medial superior frontal gyrus, and bilateral hippocampus. We also found the right inferior OFC was active regardless of aesthetic valence, and the right putamen was active for beauty judgments of oracle bone scripts that referred to positive social meanings. Although no activation in the inferior OFC was found in the analysis with aesthetic valence, the putamen has been strongly implicated in reward process ${ }^{19,50-53}$. As hypothesized, our results are consistent with previous neuroaesthetics findings, and could be interpreted as that beautiful judgments of oracle bone scripts referring to positive social meanings also commonly rely on perceptual, 


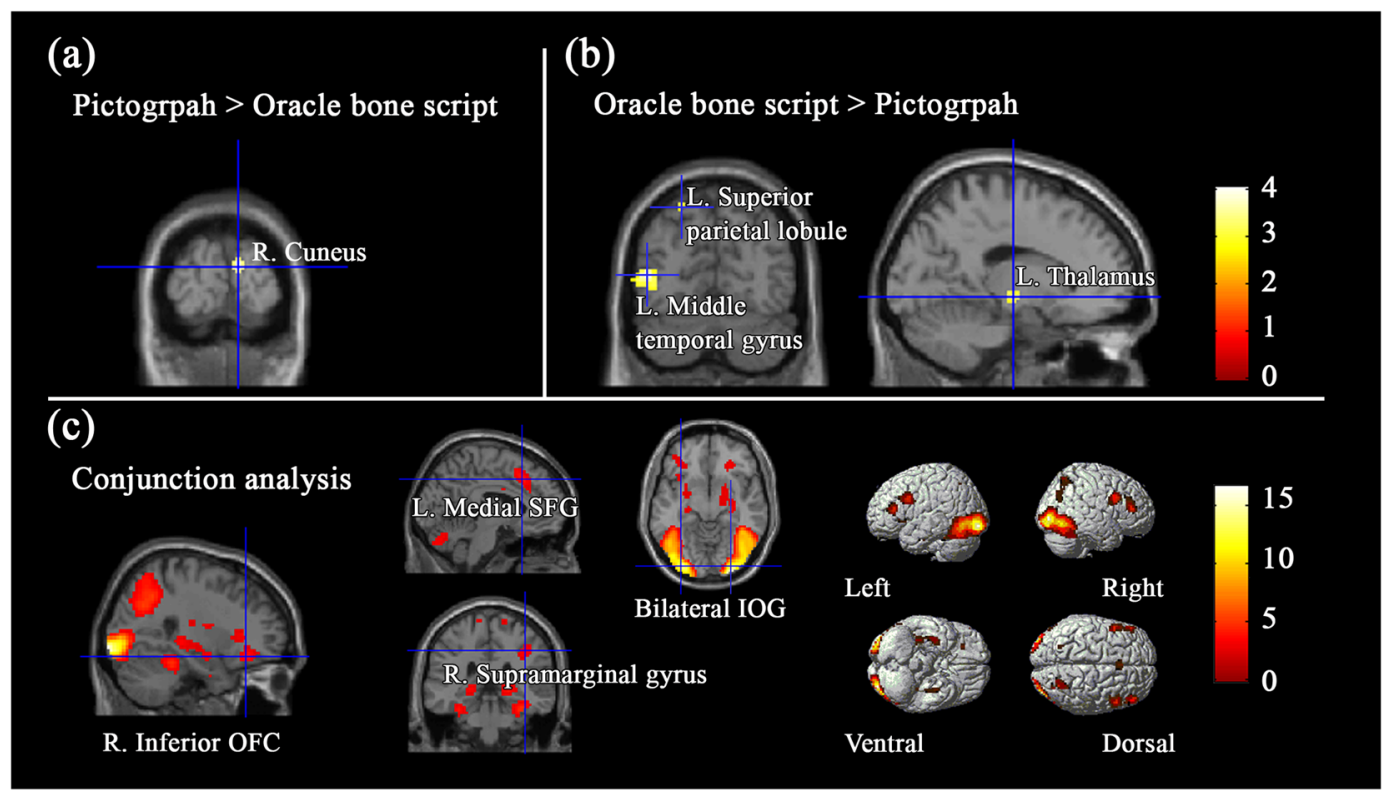

Figure 4. Cerebral areas for aesthetic judgments regardless of the aesthetic valence.

cognitive, emotional and reward processes. More importantly, the involvement of these regions clearly indicates that aesthetic appraisals of concrete pictographs and abstract oracle bone scripts alike, are not simple reactions, but rather involve multiple cognitive functions with widespread distributed neural networks ${ }^{30}$.

In contrast to judgments of beauty for pictographs, no activity in motor brain networks related to embodied approaching motivation was found when making judgments of beauty for oracle bone scripts. We propose that this absence of activation of motor areas may be attributed to low visualization demands of the oracle bone script stimuli. More specifically, pictographs were generated by outlining the external morphologies of their referential objects $^{22,23}$, making them more similar to their referential objects and having higher visualization demands than abstract oracle bone script stimuli. Hence, more perceptual representations were activated because of high visual imagery of pictographs, which facilitated the generation of embodied experience.

It is likely that only perceptual processing was engaged when deciding that pictographs and oracle bone scripts were ugly or negative; our conjunction analysis revealed common activations in bilateral inferior occipital gyri. This result replicated similar findings from studies using graphic patterns ${ }^{54}$ and pictographs ${ }^{21}$ as materials which were judged as not-beautiful or ugly, suggesting the ugly materials can be regarded as aversive stimuli ${ }^{28}$ and have an inhibitory effect on further cognitive and emotional processing.

Greater activation in the cuneus and inferior temporal gyrus was observed for pictographs than for oracle bone scripts during judgments of beauty. However, no significant activation was found in the opposite contrast. Previous studies have related the inferior temporal gyrus to visual imagery ${ }^{55}$ and the representation of object shape $^{56}$, and the cuneus to the aesthetic appreciation of beauty ${ }^{32,57}$. Thus, the stronger activation in these brain structures may be explained by a tendency for higher aesthetic preference for stimuli with high visualization demands ${ }^{10,58}$. During judgments of ugly pictographs and negative oracle bone scripts, only the contrast of "negative oracle bone script > ugly pictograph" resulted in significant activation differences, which were located in the superior temporal gyrus and fusiform gyrus. Previous studies have demonstrated that the superior temporal gyrus is involved in a more abstract aesthetic processing ${ }^{59}$ (e.g., moral beauty), and the fusiform gyrus is related to perceptual processing ${ }^{32,37}$. Therefore, it is possible that activity in these brain regions served as an extra neural resource for the aesthetic judgments of oracle bone script due to their low visualization.

To summarize, the present findings support our hypothesis that pictographs and oracle bone scripts can elicit aesthetic appraisal related to their referential objects and social meanings, respectively. Neural correlates of aesthetic appraisal of the two sets of materials suggest that judgments of beauty for pictographs and oracle bone scripts rely on common neural pathways supporting perceptual, cognitive and reward processing. Moreover, only judgments of beauty for pictographs referring to concrete objects, but not oracle bone scripts referring to abstract social meanings involved motor-related circuitry, which implies higher visualization is associated with embodied experience and approaching motivation for beauty.

Theoretically, our behavioral and neuroimaging findings provide preliminary interpretations for the mental and neural mechanisms of the two exclusive processes of beauty by using ancient Chinese characters, which showed that the aesthetic judgments of pictographs mainly depended on the aesthetic qualities of the referential objects, and involved activity in reward and motor-related areas. It indicates that beauty originates from the objective parameters and morphologies of stimuli, which can be regarded as the sense of embodied natural beauty and support the theory that "beauty has its corresponding morphological characteristics". For oracle bone scripts, the aesthetic judgments were affected by the positive degree of their social meanings, and the corresponding judgments of beauty additionally activated subcortical reward regions. This indicates that beauty is also affected by 


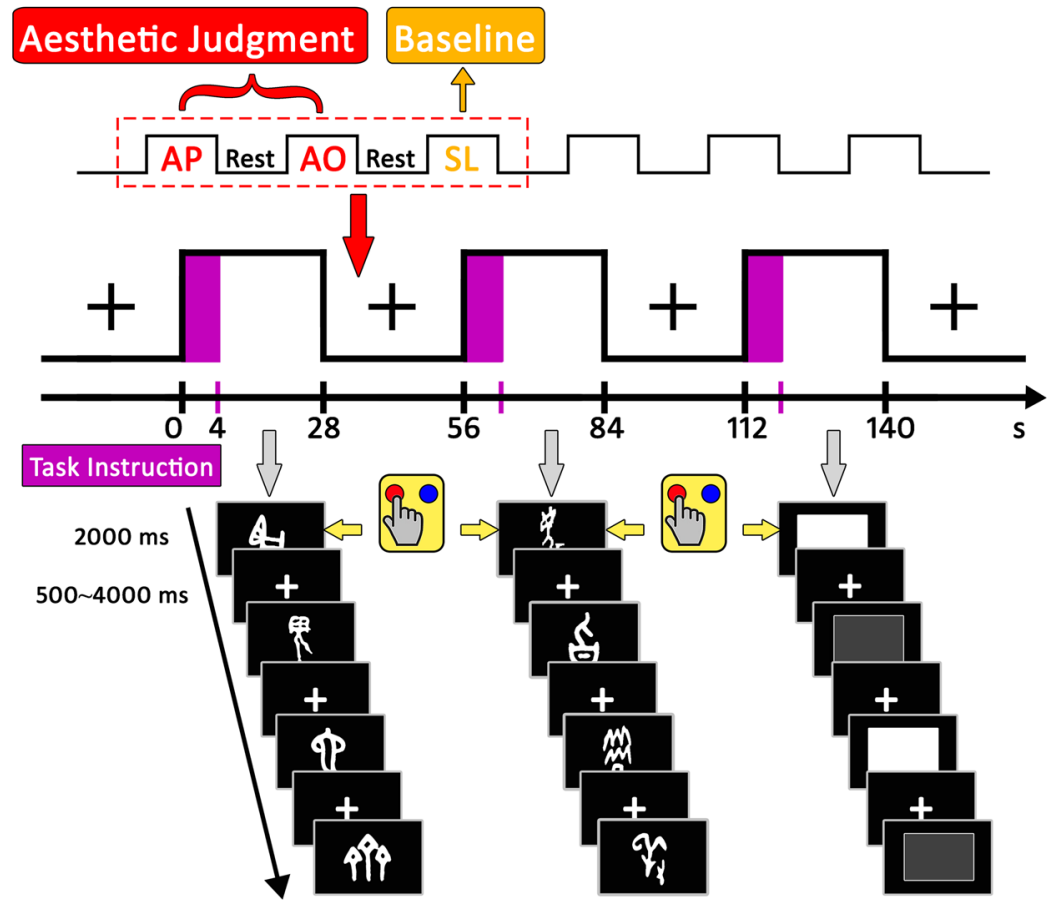

Figure 5. Experimental design, procedure and examples of stimuli. Three types of tasks were performed in separate blocks: AP judgments (beautiful pictograph vs. ugly pictograph), AO judgments (positive oracle bone script vs. negative oracle bone script), SL judgments (high luminance vs. low luminance). Examples in the first column are pictographs, referring to "rabbit", "sacrificial mask", "boa constrictor" and "seedling", from top to bottom. Examples in the second column are abstract oracle bone scripts, referring to "contemptuous", "sweet", "catastrophic" and "courteous", from top to bottom. Examples in the third column are grey squares, for high and low luminance, respectively.

subjective social construction, which can be regarded as the sense of social endowed beauty and is in accordance with the theory "what is good is beautiful, and bad is ugly".

\section{Methods}

Participants. Nineteen healthy right-handed college students (12 females) between 19 to 27 years of age (mean age $=21.74, S D=2.18$ ) gave written informed consent for this experiment, according to procedures approved by the Institute Ethics Committee, South China Normal University. None of them had received university education within any Chinese language-related majors or professional training in the arts. All participants had normal or corrected-to-normal vision, and none of them had a history of neurological or psychiatric disorders. They each received 100 yuan RMB (about \$15) for their participation. The study was design and conducted in accordance with the guidelines of the Helsinki Declaration.

Experimental procedures. Stimuli. The samples of stimuli and the experimental procedures for the present study are illustrated in Fig. 5. We used three categories of stimuli: pictographs, abstract oracle bone scripts, and grey squares. Pictographs are ancient Chinese characters that referred to concrete objects, which were adopted from the study by Zhang et al. ${ }^{20,21}$.

Abstract oracle bone scripts are ancient Chinese characters that referred to abstract social meanings. The oracle bone script meanings were based on the semantic interpretation provided by Oracle Bone Scripts Dictionary ${ }^{60}$ and were rated by a separate group of participants $(n=23)$ as to whether they reflected positive social meaning and negative social meaning. From an original pool of 98 abstract oracle bone scripts, we selected 24 that referred to positive social meanings and 24 that referred to negative social meaning. The first set of ratings presented the modern simplified two-character Chinese words corresponding each of the 98 abstract oracle bone scripts. For each word, participants rated its social meaning (positive or negative) on a 7-point scale, with 1 for extremely negative, and 7 for extremely positive. Ratings of the positive degrees of their social meanings between the two sets of experimental materials $(6.24 \pm 0.35 ; 1.86 \pm 0.49$, for positive and negative social meanings that the words referred to, respectively) were significant different, $F(1,46)=1277.09, p<0.001$.

The second set of ratings used the 98 abstract oracle bone scripts as materials. The easiness of guessing the semantic meaning of oracle bone script and the visual complexity of oracle bone script were also rated on a scale from 1 to 7, 1 for extremely hard or extremely simple, and 7 for extremely easy or extremely complicated. The rating results showed no significant differences in terms of the easiness of guessing the semantic meanings of oracle bone scripts $(1.53 \pm 0.29 ; 1.37 \pm 0.39$, for oracle bone scripts referring to positive and negative social meanings, respectively), $F(1,46)=2.59, p=0.115$. There were also no significant differences in terms of the complexity 
$(4.36 \pm 0.79 ; 4.71 \pm 0.95$, for oracle bone scripts referring to positive and negative social meanings, respectively), $F(1,46)=1.96, p=0.168$.

For grey squares, we adopted 24 high luminance grey squares $(\mathrm{RGB}=255,255,255)$ and 24 low luminance grey squares $(\mathrm{RGB}=64,64,64)$ from the study by Zhang et al. ${ }^{21}$.

The pictographs and abstract oracle bone scripts were presented in a 100-point white font. Grey squares were adjusted to be equal in size within a rectangular 'window' sized $300 \times 200$ pixels. All the three types of experimental stimuli were centered on a $600 \times 400$ pixel black background and presented at a screen resolution of $800 \times 600$ pixels.

Task. Participants performed two kinds of tasks during the scanning. In the aesthetic judgment task, participants were instructed to judge if the pictograph (block AP) or oracle bone script character (block AO) was beautiful or ugly by pressing one of two buttons with different hands. The square luminance judgment task served as a baseline task, similar to the studies by Tsukiura \& Cabeza ${ }^{18}$ and Zhang et al. ${ }^{21}$. Participants were instructed to judge whether the luminance of the square (block SL) was high or low by pressing one of two buttons. The finger-response mapping was counterbalanced across participants.

In order to avoid participants knowing or being familiar with the semantic meaning of stimuli, each participant rated the easiness of guessing the semantic meanings of pictographs and oracle bone scripts which were used in the experiment outside the MR-scanner by using a 7-point scale, with 1 for very hard, and 7 for very easy.

Procedure. The scanning session used a hybrid design with 16 blocks pertaining of each of the three conditions, AP, AO, and SL. Each condition included 48 stimuli each repeated once, resulting in a total of 96 trials. Participants underwent 4 separate scanner runs; each run consisted of 12 blocks. Block order was fixed and counterbalanced across participants. Each block contained 6 trials and lasted for $28 \mathrm{~s}$. There was a $28 \mathrm{~s}$ fixation interval between blocks. At the beginning of each block, a $4 \mathrm{~s}$ visual task instruction informed the participants about task to be performed in the upcoming block. On each trial the stimulus was presented in the center of the screen for $2 \mathrm{~s}$ including response time in pseudo-random order (event-related design), and was followed by a jittered 500-4000 ms inter-stimulus interval (ISI).

Data acquisition. Anatomical T1-weighted and functional T2*-weight MR images were conducted on a 3-Tesla Siemens Trio Tim MRI scanner, using a 12-channel phased-array head coil at the Magnetic Resonance Imaging Lab, South China Normal University. Functional images were acquired using a T2*-weighted gradient echo, echo-planar imaging sequence (32 axial slices covering the whole brain, $\mathrm{TR}=2000 \mathrm{~ms}$, $\mathrm{TE}=30 \mathrm{~ms}$, flip angle $=90^{\circ}, \mathrm{FOV}=192 \mathrm{~mm}$, inter-slice gap $=1 \mathrm{~mm}$, slice thickness $=3 \mathrm{~mm}$, matrix size $\left.=64 \times 64\right)$. After the functional scanning, a high-resolution T1-weighted anatomical scan was acquired by using a MP-RAGE sequence $\left(\mathrm{TR}=1900 \mathrm{~ms}, \mathrm{TE}=2.52 \mathrm{~ms}\right.$, flip angle $=9^{\circ}$, voxel-size $\left.=1 \mathrm{~mm} \times 1 \mathrm{~mm} \times 1 \mathrm{~mm}\right)$.

Data analysis. Image pre-processing and analysis were performed using SPM8 (http://www.fil.ion.ucl.ac.uk/ $\mathrm{spm} /$ ). The first 6 volumes of each functional run were discarded to allow for T1 equilibration. All remaining volumes from each participant were preprocessed with slice-scan time correction, spatially realigned to the first volume for correcting head movements, coregistered to the T1-weighted structural image, normalized to the standard template based on the MNI reference brain, resampled with voxel size of $3 \times 3 \times 3 \mathrm{~mm}^{3}$, and spatially smoothed with an isotropic $8 \mathrm{~mm}$ full width-half-maximum (FWHM) Gaussian kernel. Two participants were excluded in the subsequent analysis as one individual image had $>2 \mathrm{~mm}$ maximum displacement and $>1.5^{\circ}$ rotation, and one individual had low accuracy rates for the aesthetic judgments.

At the first (single participant) level, a general linear model (GLM) was applied to the time-series data, in which stimulus onset was modeled as a single impulse response function, and then convolved with the canonical haemodynamic response function (HRF). We modeled six regressors of interest: pictograph referring to beautiful object $(\mathrm{PB})$, pictograph referring to ugly object $(\mathrm{PU})$, oracle bone script referring to positive social meaning $(\mathrm{OP})$, oracle bone script referring to negative social meaning $(\mathrm{ON})$, high luminance $(\mathrm{HL})$ and low luminance (LL). Head movement parameters calculated from the realignment procedure were included as regressors of no interest. Low frequency drifts were removed using a high-pass filter with a cut-off of $128 \mathrm{~s}$. The resulting contrast images for each condition were entered into the second level (random-effects) analyses in which the regressors of interest were modeled using Flexible Factorial analyses. In order to identify the cortical networks involved in the aesthetic judgments of pictographs and oracle bone scripts we utilized the square luminance judgment as baseline to control for activity in motor brain regions associated with the key responses. Therefore, we first performed the contrasts of "PB $>$ HL", "PU $>$ LL" "OP $>$ HL" and "ON $>$ LL". Based on the four contrasts, we computed a conjunction between the "PB $>$ HL" and "OP $>$ HL", "PU $>$ LL" and "ON $>$ LL" using the minimum statistic approach ${ }^{61}$. Moreover, direct comparison of "PB $>$ OP", "PU $>$ ON", "OP $>$ PB" and "ON $>$ PU" were conducted to investigate differences in neural mechanisms between aesthetic judgments of pictographs and oracle bone scripts. Statistical thresholds for Flexible Factorial analyses were set at $p<0.001$ at voxel level (uncorrected), $p<0.05$ at cluster level (FWE corrected).

\section{References}

1. Di Dio, C., Macaluso, E. \& Rizzolatti, G. The golden beauty: Brain response to classical and renaissance sculpture. PLoS ONE. 2(11), e1201, doi:10.1371/journal.pone.0001201 (2007).

2. Koehler, N., Rhodes, G. \& Simmons, L. W. Are human female preferences for symmetrical male faces enhanced when conception is likely? Anim. Behav. 64, 233-238 (2002).

3. Little, A. C., Jones, B. C., DeBruine, L. M. \& Feinberg, D. F. Symmetry and sexual dimorphism in human faces: Interrelated preferences suggest both signal quality. Behav. Ecol. 19, 902-908 (2008) 
4. Rhodes, G. et al. Attractiveness of facial averageness and symmetry in non-Western cultures: In search of biologically based standards of beauty. Perception. 30, 611-625 (2001).

5. Jacobsen, T., Schubotz, R. I., Höfel, L. \& von Cramon, D. Y. Brain correlates of aesthetic judgment of beauty. NeuroImage. 29, 276-285 (2006)

6. Seckler, M., Opwis, K. \& Tuch, A. N. Linking objective design factors with subjective aesthetics: An experimental study on how structure and color of websites affect the facets of users' visual aesthetic perception. Comput. Hum. Behav. 49, 375-389 (2015).

7. Orgs, G., Hagura, N. \& Haggard, P. Learning to like it: Aesthetic perception of bodies, movements and choreographic structure. Conscious. Cogn. 22, 603-612 (2013).

8. Cattaneo, Z. et al. The role of the lateral occipital cortex in aesthetic appreciation of representational and abstract paintings: A TMS study. Brain. Cogn. 95, 44-53 (2015).

9. Cusack, P., Lankston, L. \& Isles, C. Impact of visual art in patient waiting rooms: Survey of patients attending a transplant clinic in Dumfries. JRSM Short. Rep. 1, 52, doi:10.1258/shorts.2010.010077 (2010).

10. Vessel, E. A. \& Rubin, N. Beauty and the beholder: Highly individual taste for abstract, but not real-world images. J. Vis. 10, 1-14 (2010).

11. Hekkert, P. \& van Wieringen, P. C. W. Complexity and prototypicality as determinants of the appraisal of cubist paintings. Brit. J. Psychol. 81, 483-495 (1990).

12. Martindale, C., Moore, K. \& Borkum, J. Aesthetic preference: Anomalous findings for Berlyne's psychobiological theory. Am. J. Psychol. 103, 53-80 (1990).

13. O'Doherty, J. et al. Beauty in a smile: The role of medial orbitofrontal cortex in facial attractiveness. Neuropsychologia. 41, 147-155 (2003).

14. Kampe, K. K. W., Frith, C. D., Dolan, R. J. \& Frith, U. Reward value of attractiveness and gaze. Nature. 413, 589 (2001).

15. Gross, A. E. \& Crofton, C. What is good is beautiful. Sociometry. 40, 85-90 (1977).

16. Hassin, R. \& Trope, Y. Facing faces: Studies on the cognitive aspects of physiognomy. J. Pers. Soc. Psychol. 78, 837-852 (2000).

17. Owens, G. \& Ford, J. G. Further consideration of the "What is good is beautiful" finding. Soc. Psychol. 41, 73-75 (1978).

18. Tsukiura, T. \& Cabeza, R. Shared brain activity for aesthetic and moral judgments: Implications for the Beauty-is-Good stereotype. Soc. Cogn. Affect. Neurosci 6, 138-148 (2011)

19. Wang, T. T. et al. Is moral beauty different from facial beauty? Evidence from an fMRI study. Soc. Cogn. Affect. Neurosci. 10, 6, doi:10.1093/scan/nsu123 (2014).

20. Zhang, W. et al. The embodiment of beauty: Evidence from viewing Chinese concrete words and pictographs. Int. J. Psychol. doi:10.1002/ijop.12269 (2016).

21. Zhang, W., Lai, S., He, X., Zhao, X. \& Lai, S. Neural correlates for aesthetic appraisal of pictograph and its referent: An fMRI study. Behav. Brain. Res. 305, 229-238 (2016).

22. Guo, M. R. The dialectic development of ancient Chinese characters. Chin. J. Archaeol 1, 1-13 (1972).

23. Li, Y. F. Re-understanding of the properties and academic value of Liùshū. Chin. Teach. World. 26(1), 94-105 (2012).

24. Chatterjee, A. Neuroaesthetics: A coming of age story. J. Cogn. Neurosci. 23, 53-62 (2011).

25. Chatterjee, A. \& Vartanian, O. Neuroaesthetics. Trends. Cogn. Sci. 18, 370-375 (2014).

26. Nadal, M. \& Skov, M. Introduction to the special issue: Toward an interdisciplinary neuroaesthetics. Psychol. Aesthet. Creativity. Arts. 7, 1-12 (2013).

27. Gibbs, R. W. Jr., Costa Lima, P. L. \& Francozo, E. Metaphor is grounded in embodied experience. J. Pragmat. 36, 1189-1210 (2004).

28. Kawabata, H. \& Zeki, S. Neural correlates of beauty. J. Neurophysiol. 91, 1699-1705 (2004).

29. Koch, S. C. Arts and health: Active factors and a theory framework of embodied aesthetics. Arts Psychother. 54, 85-91 (2017).

30. Boccia, M. et al. Where does brain neural activation in aesthetic responses to visual art occur? Meta-analytic evidence from neuroimaging studies. Neurosci. Biobehav. Rev. 60, 65-71 (2016).

31. Cela-Conde, C. J. et al. Activation of the prefrontal cortex in the human visual aesthetic perception. Proc. Natl. Acad. Sci. USA 101, 6321-6325 (2004).

32. Cupchik, G. C., Vartanian, O., Crawley, A. \& Milkulis, D. J. Viewing artworks: Contributions of cognitive control and perceptual facilitation to aesthetic experience. Brain. Cogn. 70, 84-91 (2009).

33. Avram, M. et al. Neurofunctional correlates of esthetic and moral judgments. Neurosci. Lett. 534, 128-132 (2013).

34. Bzdok, D. et al. Parsing the neural correlates of moral cognition: ALE meta-analysis on morality, theory of mind, and empathy. Brain. Struct. Funct. 217, 783-796 (2012).

35. Moll, J. et al. The self as a moral agent: Linking the neural bases of social agency and moral sensitivity. Soc. Neurosci. 2, 336-352 (2007)

36. Blood, A. J. \& Zatorre, R. J. Intensely pleasurable responses to music correlate with activity in brain regions implicated in reward and emotion. Proc. Natl. Acad. Sci. USA 98, 11818-11823 (2001).

37. Bohrn, I. C., Altmann, U., Lubrich, O., Menninghaus, W. \& Jacobs, A. M. When we like what we know-A parametric fMRI analysis of beauty and familiarity. Brain. Lang. 124, 1-8 (2013).

38. Bradley, M. M. et al. Imaging distributed and massed repetitions of natural scenes: Spontaneous retrieval and maintenance. Hum. Brain. Mapp. 36, 1381-1392 (2015).

39. Ito, T. et al. Changing the mind? Not really-activity and connectivity in the caudate correlates with changes of choice. Soc. Cogn. Affect. Neurosci. 9, 1546-1551 (2014).

40. Brown, S., Gao, X., Tisdelle, L., Eickhoff, S. B. \& Liotti, M. Naturalizing aesthetics: Brain areas for aesthetic appraisal across sensory modalities. Neuroimage. 58, 250-258 (2011).

41. Pessoa, L. On the relationship between emotion and cognition. Nat. Rev. Neurosci. 9, 148-158 (2008)

42. Rolls, E. T. \& Grabenhorst, F. The orbitofrontal cortex and beyond: From affect to decision-making. Prog. Neurobiol. 86, 216-244 (2008).

43. Cela-Conde, C. J. et al. Sex-related similarities and differences in the neural correlates of beauty. Proc. Natl. Acad. Sci. USA 106, 3847-3852 (2009).

44. Di Dio, C. \& Gallese, V. Neuroaesthetics: A review. Curr. Opin. Neurobiol. 19, 682-687 (2009).

45. Moore, C. I. et al. Segregation of somatosensory activation in the human rolandic cortex using fMRI. J. Neurophysiol. 84, 558-569 (2000).

46. Rushworth, M. F. S., Krams, M. \& Passingham, R. E. The attentional role of the left parietal cortex: The distinct lateralization and localization of motor attention in the human brain. J. Cogn. Neurosci. 13, 698-710 (2001).

47. Amiez, C. \& Petrides, M. Neuroimaging evidence of the anatomo-functional organization of the human cingulate motor areas. Cereb. Cortex. 24, 563-578 (2014).

48. Havel, P. et al. Reproducibility of activation in four motor paradigms: An fMRI study. J. Neurol. 253, 471-476 (2006).

49. Margulies, D. S. Precuneus shares intrinsic functional architecture in humans and monkeys. Proc. Natl. Acad. Sci. USA 106, 20069-20074 (2009).

50. Carretié, L. et al. The striatum beyond reward: Caudate responds intensely to unpleasant pictures. Neuroscience. 164, 1615-1622 (2009).

51. Sailer, U. et al. Temporal dynamics of brain activation during 40 minutes of pleasant touch. NeuroImage. 139, 360-367 (2016) 
52. Tsukiura, T. \& Cabeza, R. Remembering beauty: Roles of orbitofrontal and hippocampal regions in successful memory encoding of attractive faces. Neuroimage. 54, 653-660 (2011).

53. Vicente, A. F., Bermudez, M. A., Romero, M. D. C., Perez, R. \& Gonzalez, F. Putamen neurons process both sensory and motor information during a complex task. Brain. Res. 1466, 70-81 (2012).

54. Jacobsen, T. \& Höfel, L. Descriptive and evaluative judgment processes: Behavioral and electrophysiological indices of processing symmetry and aesthetics. Cogn. Affect. Behav. Neurosci. 3, 289-299 (2003).

55. Hayashi, A. et al. Visual imagery while reading concrete and abstract Japanese kanji words: An fMRI study. Neurosci. Res. 79, 61-66 (2014).

56. Zwaan, R. A. \& Yaxley, R. H. Lateralization of object-shape information in semantic processing. Cognition. 94, B35-B43 (2004).

57. Mizokami, Y. et al. Difference in brain activations during appreciating paintings and photographic analogs. Front. Hum. Neurosci. 8, 478, doi:10.3389/fnhum.2014.00478 (2014).

58. Pihko, E. et al. Experiencing art: The influence of expertise and painting abstraction level. Front. Hum. Neurosci. 5, 94, doi:10.3389/ fnhum.2011.00094 (2011).

59. Boccia, M. et al. Neural foundation of human moral reasoning: an ALE meta-analysis about the role of personal perspective. Brain. Imaging. Behav. 10.1007/s11682-016-9505-x (2016).

60. Meng, S. K. Oracle Bone Scripts Dictionary (Shanghai People’s Publishing House, 2009).

61. Nichols, T., Brett, M., Andersson, J., Wager, T. \& Poline, J. B. Valid conjunction inference with the minimum statistic. NeuroImage. 25, 653-660 (2005)

\section{Acknowledgements}

The authors warmly thank Professor Carol Seger for proof reading and editing the manuscript. This work was supported by the Natural Science Foundation of China (grant number 31671132).

\section{Author Contributions}

The contributions of the first and the second author to this paper are equal. Wei Zhang and Xianyou He designs the experiments and drafted the article; Xianyou He also revised this manuscript critically; Siyan Lai and Juan Wan for data pre-processing and analysis; Shuxian Lai, Xueru Zhao for data collection and making experimental materials; Darong Li contributed to the revised version of the manuscript.

\section{Additional Information}

Competing Interests: The authors declare that they have no competing interests.

Publisher's note: Springer Nature remains neutral with regard to jurisdictional claims in published maps and institutional affiliations.

(c) (i) Open Access This article is licensed under a Creative Commons Attribution 4.0 International Cicense, which permits use, sharing, adaptation, distribution and reproduction in any medium or format, as long as you give appropriate credit to the original author(s) and the source, provide a link to the Creative Commons license, and indicate if changes were made. The images or other third party material in this article are included in the article's Creative Commons license, unless indicated otherwise in a credit line to the material. If material is not included in the article's Creative Commons license and your intended use is not permitted by statutory regulation or exceeds the permitted use, you will need to obtain permission directly from the copyright holder. To view a copy of this license, visit http://creativecommons.org/licenses/by/4.0/.

(C) The Author(s) 2017 